\title{
Correction to: Three-dimensional soft tissue changes according to skeletal changes after mandibular setback surgery by using cone-beam computed tomography and a structured light scanner
}

\author{
Kyung-A Kim', Ye-Jin Chang ${ }^{2}$, Su-Hyun Lee ${ }^{2}$, Hyun-Joon $\mathrm{An}^{2}$ and Ki-Ho Park ${ }^{1 *}$ (D)
}

Correction to: Prog Orthod (2019) 20:25

https://doi.org/10.1186/s40510-019-0282-0

In the publication of this article [1], there are errors in the Methods (Subjects) and Ethics approval and consent to participate section.

The error:

"This retrospective study was approved by the Institutional Review Board of XXXXX XXX University Medical Center (IRB No: K-2013-11018794). Skeletal Class III patients who had received bilateral sagittal split ramus osteotomy only by the same surgeon at the XXXXX XXX University Dental Hospital had been screened."

Should instead read:

"This retrospective study was approved by the Institutional Review Board of Kyung Hee University Medical Center (IRB No: K-2013-11018794). Skeletal Class III patients who had received bilateral sagittal split ramus osteotomy only by the same surgeon at the Kyung Hee University Dental Hospital had been screened."
The error:

"This retrospective study was approved by the Institutional Review Board of XXXXX XXX University Medical Center (IRB No: K-2013-11018794)."

Should instead read:

"This retrospective study was approved by the Institutional Review Board of Kyung Hee University Medical Center (IRB No: K-2013-11018794)."

This has now been updated in the original article [1].

\section{Author details}

${ }^{1}$ Department of Orthodontics, School of Dentistry, Kyung Hee University, 1 Hoegi-Dong, Dongdaemoon-Ku, Seoul 130-701, South Korea. ${ }^{2}$ Department of Orthodontics, Graduate School, Kyung Hee University, Seoul, South Korea.

Published online: 26 July 2019

\section{Reference}

1. Kim KA, Chang YJ, Lee SH, et al. Three-dimensional soft tissue changes according to skeletal changes after mandibular setback surgery by using cone-beam computed tomography and a structured light scanner. Prog Orthod. 2019;20:25. https://doi.org/10.1186/s40510-019-0282-0.

\footnotetext{
* Correspondence: pkhmate@hanmail.net

The original article can be found online at https://doi.org/10.1186/s40510-01 9-0282-0

'Department of Orthodontics, School of Dentistry, Kyung Hee University, 1 Hoegi-Dong, Dongdaemoon-Ku, Seoul 130-701, South Korea

Full list of author information is available at the end of the article
} 